\title{
Inner ear damage from toy cap pistols and fire-crackers
}

\author{
Alf Axelsson ${ }^{1}$, Per-Anders Hellström ${ }^{1}$, Richard Altschuler ${ }^{2}$ \\ and Josef M. Miller ${ }^{2}$ \\ 'Department of Audiology, The University of Göteborg, Sahlgrenska Hospital, Göteborg (Sweden) \\ and ${ }^{2}$ Kresge Hearing Research Institute, The University of Michigan, Ann Arbor, MI 48109-0506 (U.S.A.) \\ (Received 6 April 1990) \\ (Revised version received 26 November 1990) \\ (Accepted 3 December 1990)
}

Key words: Guinea pig; Noisy toy; Impulsive noise; Sound level; Noise-induced hearing loss

\begin{abstract}
Groups of guinea pigs comprising 7 animals in each group were exposed to 10,50 or 100 exposures to fire-crackers or 10,50 or 100 exposures to toy cap pistol shots. An additional group of 7 animals comprised the control material. The exposures were performed with $15-\mathrm{s}$ intervals at $0.25 \mathrm{~m}$ distance for the toy cap pistol shots and at $0.8 \mathrm{~m}$ for the fire-crackers. The peak sound level at the ear was $155 \mathrm{dBC}$ for both impulsive sounds. After a 3-week survival period the animals were anesthetized and decapitated. The cochleas were examined histologically in surface preparations and read double-blind. One animal in each group exposed to 10 fire-crackers and 10 toy cap pistol shots showed sensory cell loss. With 50 or 100 toy cap pistol shots or fire-cracker exposures, 24 out of 28 animals showed pronounced sensory cell loss. The present results clearly indicate the risk for noise-induced hearing loss in children playing with toy cap guns and fire-crackers.
\end{abstract}

\section{Introduction}

Previous investigations have shown that teenage boys frequently have a slight sensorineural high-tone hearing loss similar to that expected from exposure to high levels of noise $[2,3,7,11]$. This hearing loss, which can be found in $15 \%$ of 16 -year-old

Correspondence: A. Axelsson, Department of Audiology. University of Göteborg. Sahlgren's Hospita!. S-413 45 Göteborg, Sweden. 
boys, might be due to genetic causes, noisy games and toys, or a combination of the two. It is well known that these 'noise dips', occur more commonly in boys than in girls. It has been suggested that the typically more agressive behavior and noisy games of boys, compared to girls, supports the notion that high-intensity sound exposure contributes to this hearing loss $[1,9]$.

Some of the toys that boys use emit impulsive sound, such as fire-crackers and toy cap guns. Sound levels from such toys have been shown to be very high $[2,4-6,8,10]$. The damage from sound on the sensitive sensory cells of the inner ear depends on the intensity and the duration of the sound. Surprisingly, many of these toys emit impulsive sounds where the intensities exceed the established damage risk criteria for hearing loss (140 dB peak level). However, the duration of these impulses is very short; typically microseconds or a few milliseconds and the apparent loudness of a sound decreases with durations of less than $25 \mathrm{~ms}$. Thus even though an impulse sound may be at a level which can cause permanent damage to the inner ear, it may not sound particularly loud. Changes in hearing from exposure to fire-crackers and toy cap pistols have been quite variable, probably due to variability in the exposure conditions. Few well-controlled evaluations of the potential damage resulting from these toys have been performed. In one report [8] there was extensive loss of hair cells in guinea pigs exposed to only one toy cap pistol shot.

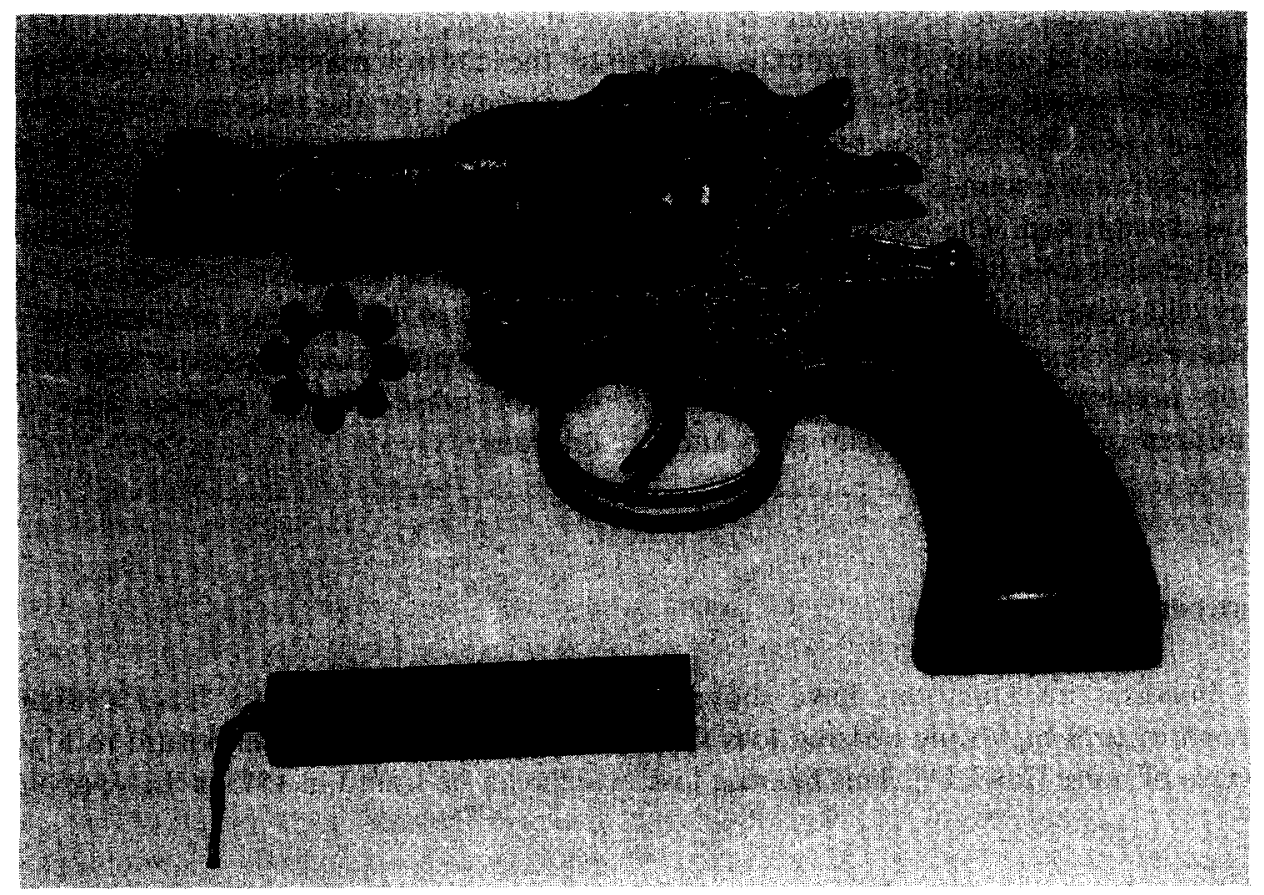

Fig. 1. The toy cap pistol and the fire-cracker used in the present investigation. 
In order to investigate the relationship between impulsive sounds from toy cap pistols and fire-crackers (Fig. 1), we examined the influence of these toys on the histopathology of the inner ear in guinea pigs using histological methods.

\section{Materials and methods}

Forty-eight 4-week-old guinea pigs were divided into 7 groups (Table I). The experimental animals were un-anesthetized, wrapped in a towel with their nose facing the sound source and both ears were exposed. The subjects were positioned in a semicircle about the toy cap pistol and fire-crackers sound source (Fig. 2) in the middle of an anechoic chamber on a steel-wire netting mounted horizontally. The exposures were performed manually at 15 -s intervals except for two fire-crackers where the intervals were 25 or $35 \mathrm{~s}$ due to mis-firing. The distance between the animals and the exposure was set to $0.25 \mathrm{~m}$ for the toy cap pistol and $0.8 \mathrm{~m}$ for the fire-crackers. With these distances the animals were exposed to peak levels of 155 $\mathrm{dBC}$ for both types of sounds. The peak level of each shot was registered with a Brüel and Kjaer (B\& K) 1/4" microphone 4135 connected to a B \& K sound level meter 2209 and recorder 2307. Animals from the control group were also placed in an anechoic room under similar conditions for $25 \mathrm{~min}$ but not exposed to noise. However, there was still some smoke from the fire-crackers in the chamber. After the noise exposure the animals were returned to the animal facilities where they were kept under normal laboratory conditions for 3 weeks.

After the 3-week survival period the animals were anesthetized and decapitated. The temporal bones were removed, the bulla opened and a small hole made in the apex and round window of the cochlea. The cochleas were fixed by slow injection of 2.5\% glutaraldehyde and immersed in the fixative for $24 \mathrm{~h}$. Subsequently. the animals were processed using surface preparation techniques for hair cell counts.

TABLE I

Present material

\begin{tabular}{lccc}
\hline Sound source & n animals & n exposures & $\begin{array}{l}\text { Interval } \\
\text { between } \\
\text { exposures }\end{array}$ \\
\hline Control & 7 & - & - \\
Toy cap gun & 7 & $10 \times$ & $15 \mathrm{~s}$ \\
Toy cap gun & 7 & $50 \times$ & $15 \mathrm{~s}$ \\
Toy cap gun & 7 & $100 \times$ & $15 \mathrm{~s}$ \\
Fire-cracker & 6 & $10 \times$ & $15 \mathrm{~s}$ \\
Fire-cracker & 7 & $100 \times$ & $15 \mathrm{~s}$ \\
Fire-cracker & 7 & & $15 \mathrm{~s}$ \\
& 48 & & \\
\hline
\end{tabular}

* 2 out of 50 mis-fired-interval 25 and $35 \mathrm{~s}$. 


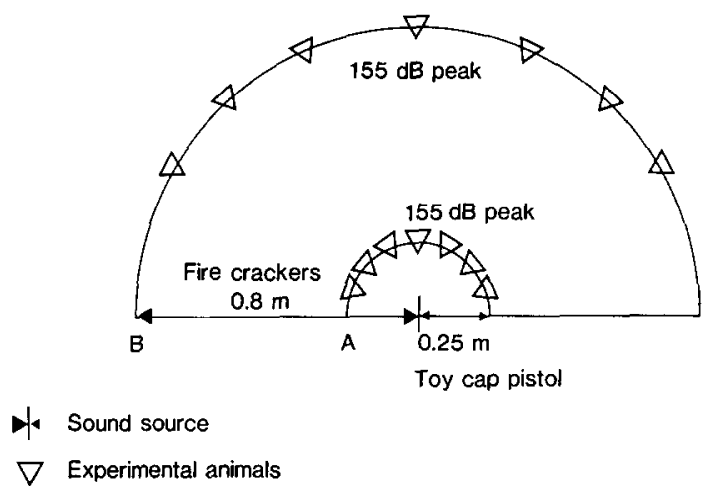

Fig. 2. Schematic drawing of the exposure situation with the experimental animals at $25 \mathrm{~cm}$ (toy cap pistol) or $80 \mathrm{~cm}$ (fire-crackers) from the sound source.

Only missing outer or inner hair cells were counted. Minor changes such as swollen cells or stereocilia disarray was not considered as sensory cell loss. The animals were read blind so that the reader was unaware of what experimental group a given animal belonged to.

\section{Results}

The histological changes were graded into normal, i.e. less than $10 \%$ scattered changes, slight sensory cell loss and pronounced sensory cell loss (more than $75 \%$ missing hair cells). In Fig. 3 it can be seen that only one of the 7 control guinea pigs showed slight damage to sensory cells. With 10 pistol shots or fire-cracker explosions most experimental animals were not significantly different from control subjects. One animal exposed to 10 toy cap pistol firings showed slight sensory cell

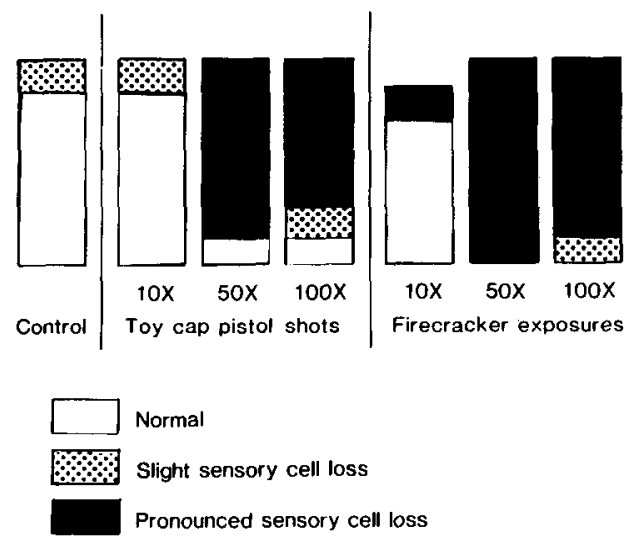

Fig. 3. The amount of sensory cell loss in relation to the number of exposures. 
loss and one animal exposed to 10 fire-crackers showed a pronounced sensory cell loss. With 50 or 100 exposures to toy cap pistol firings, 11 out of 14 animals showed pronounced sensory cell loss. However, even with this exposure, one animal in each group showed only small changes and was considered to be within normal limits. With 50 or 100 fire-crackers, 13 out of 14 animals showed pronounced sensory cell loss, and the remaining subject showed a slight sensory cell loss. The average hair cell loss in each group is significantly different from the control group $(\mathrm{P}<0.05)$, except for the 10 toy cap pistol shots group.

The detailed description of the changes in relation to distance from the apical end of the cochlea will be presented elsewhere. In the group of 7 animals exposed to 50 fire-crackers, most of the changes were localized to the area corresponding to $500-1000 \mathrm{~Hz}$. In 4 of the 7 animals the sensory cell damage extended at least to an area corresponding to $2 \mathrm{kHz}$. However, generally the distribution along the basilar membrane was quite consistent.

\section{Discussion}

The present report clearly shows that a realistic number of toy cap pistol shots or fire-cracker explosions (i.c. 50-100 exposures), within a distance from the ear that can occur during play and holidays when these toys are used, such as New Year's Eve, 4th of July in the United States or Easter and New Year in Sweden. The interval between successive exposures, particularly for the fire-crackers, may be somewhat shorter than typical. However, it is within realistic limits. We may expect that with longer intervals between exposures the amount of pathology may be somewhat reduced. Obviously, it is difficult to draw conclusions about the sensitivity for these types of impulsive sounds from guinea pigs with respect to humans. However, the present dramatic changes in the cochlea clearly show that under certain circumstances such toys can cause permanent damage to inner ear structures and irreversible sensory cell loss. These findings are consistent with clinical experience in which it is common to find that individuals with a high-tone sensorineural hearing loss can vividly remember acoustic accidents, e.g., where a fire-cracker or toy cap pistol exploded close to their ear, resulting in tinnitus and at least a temporary hearing loss. Finally, even if the use of these toys do not regularly cause inner ear damage, there is reason to believe that repeated exposures to such impulsive sounds diminish the reserve function of the inner ear which then yields a more vulnerable ear to noise, possibly more sensitive to future occupational noiseinduced hearing loss or to a loss from leisure time noise activities.

\section{References}

1 Axelsson, A., Jerson, T. and Lindgren, F., Noisy leisure time activities in teenage boys, Am. Industr. Hyg. Assoc. J., 3., 42 (1981) 229-233. 
2 Axelsson, A. and Jerson, T., Noisy toys: a possible source of sensori-neural hearing loss, Pediatrics, 4 (1985) 574-578.

3 Axelsson, A., Aniansson, G. and Costa, O.A., Hearing loss in school children, Scand. Audiol., 16 (1987) 137-143.

4 Gjaevenes, K., Measurements on the impulsive noise from crackers and toy firearms, J. Acoust. Soc. Am., 39 (1966) 403-404.

5 Gjaevenes, K., Moseng, J. and Nordahl, T., Hearing loss in children caused by the impulsive noise of chinese crackers, Scand. Audiol., 3 (1974) 153-156.

6 Marshall, L. and Brandt, J.F., Temporary threshold shift from a toy cap gun, J. Speech Hear. Disord., 39 (1974) 163-169.

7 Mills, J.H., Noise and children: a review of the literature, J. Acoust. Soc. Am., 58 (1975) 767-779.

8 Robertson, D., Cody, A.R. and Johnstone, B.M., Inner ear damage caused by a toy cap pistol, J. Otolaryng. Soc. Austral., 80 (1979) 285-288.

9 Rytzner, B. and Rytzner, C., School children and noise, Scand. Audiol., 10 (1981) 213-215.

10 Ward, W.D. and Glorig, A., A case of fire-cracker-induced hearing loss, Laryngoscope, 71 (1961) $1590-1596$.

11 Weber, H.J., Mac Govern, F.J. and Zink, D., An evaluation of 1000 children with hearing loss, J. Speech Hear. Disord., 32 (1967) 343-354. 